\title{
Características de personalidad en pacientes alcohólicos mediante el cuestionario Temperament and Character Inventory (TCl). Diferencias según la existencia de abuso de benzodiacepinas (BZD) y trastornos de la personalidad
}

\author{
Miquel Monras*; Silvia Mondon**; JoAn Jou*** \\ * Psicólogo Adjunto. \\ ** Médico Psiquiatra Adjunto. \\ *** Psicólogo Becario. \\ Unidad de Alcohologia. Instituto de Neurociencias. Hospital Clínico de Barcelona. España. \\ Enviar correspondencia a: \\ Miquel Monras Arnau. Unidad de Alcohología. Hospital Clínic de Barcelona. C/Villarroel, 170. 08036 Barcelona
}

Teléfono: (+34) 93.227.99.23. Fax: (+34) 93.227.56.88. e-mail: mmonras@clinic.ub.es

Recibido: Noviembre de 2007

Aceptado: Febrero de 2008

\section{RESUMEN}

Objetivos: Se pretende hallar características de personalidad diferenciales mediante el cuestionario TCl entre pacientes alcohólicos con o sin abuso de benzodiazepinas (BZD) o trastorno de la personalidad (TP) para convertirlos en criterios de mal o buen pronóstico y poder usarlos como dianas psicoterapéuticas. Metodología: Estudio transversal de 218 pacientes alcohólicos ingresados para desintoxicación, comparando las puntuaciones $\mathrm{TCl}$ entre abusadores o no de BZD y entre pacientes con TP o sin él. Resultados: los pacientes con abuso de BZD tienen mayor puntuación en Evitación del daño y menor en Autodirección que los no abusadores. Los pacientes con TP presentan mayor puntuación en Búsqueda de la Novedad y menor en Cooperación que los pacientes sin TP. Conclusiones: Los pacientes abusadores de BZD son menos maduros y autosuficientes, más influenciables, inseguros y muy evitadores de los riesgos. Los pacientes con TP se caracterizan por ser más impulsivos y menos cooperadores. Las características encontradas con el $\mathrm{TCl}$ son coherentes con las dificultades de estos pacientes durante el tratamiento y con las impresiones clínicas. Estas áreas problemáticas deberían convertirse en objetivos terapéuticos a modificar mediante el tratamiento. Por todo ello el TCl se revela un instrumento útil en la comprensión, evaluación e identificación de los pacientes alcohólicos y sus necesidades de tratamiento.

Palabras clave: alcoholismo, benzodiacepinas, trastorno de personalidad, temperamento, carácter.

\section{ABSTRACT}

Objective: To find differential personality traits, through the $\mathrm{TCl}$ questionnaire, between alcoholic patients who abuse benzodiazepines (BZD) and those who do not, and between patients affected by personality disorders (PD) and those not affected. These traits can then be used as criteria of good prognosis and as psychotherapeutic targets. Methods: Crosssectional study of 218 alcoholic inpatients, comparing TCl scores between BZD abusers and non-abusers, and between PD-affected patients and non-PD-affected patients. Results: BZD-abusing patients score higher in Harm Avoidance and lower in Self-Directedness than non-abusers. Patients with PD score higher in Novelty-Seeking and lower in Cooperativeness than patients without PD. Conclusions: BZD-abusing patients are less mature and self-sufficient, more impressionable, insecure, and have a high tendency to avoid risk. Patients with PD are more impulsive and less cooperative. The characteristics found with $\mathrm{TCl}$ are coherent with the difficulties of these patients during treatment and with clinical impressions. These problematic areas should become therapeutic targets to be modified through treatment. For all of these reasons the TCl emerges as a useful instrument for understanding, assessing and identifying alcoholic patients and their treatment needs.

Key words: lalcoholism, benzodiazepines, personality disorders, temperament, character. 


\section{INTRODUCCIÓN}

H oy en día se conoce la elevada prevalencia de trastornos de la personalidad que tienen los pacientes con dependencia a drogas y alcohol1, 2. También se sabe de la importante influencia que los TP tienen en la efectividad del tratamiento de sus adicciones ${ }^{3,4}$.

En este contexto últimamente está recibiendo especial atención el estudio de las características diferenciales de personalidad entre diversos tipos de pacientes psiquiátricos ${ }^{5,6}$ y respecto la población general.

Una posible utilidad de estas investigaciones es conceptual y teórica, sobretodo por la posible influencia de la personalidad en el desarrollo de una adicción.

Aunque ya está descartada la existencia de una personalidad "pretoxicómana" concreta ${ }^{7,8,9}$, son abundantes los estudios de cohorte que hallan que los rasgos antisociales ${ }^{10}$, la psicopatologia ${ }^{11}$, o la genética ${ }^{12}$, ${ }^{13}$ predicen el abuso a distintas substancias, aunque todos ellos son factores poco específicos y que interaccionan con el ambiente ${ }^{14}$.

También se ha observado que los alcohólicos, comparados con la población general, buscan más las novedades, son más individualistas, tienen mayor ansiedad anticipatoria, menos control de la conducta y son menos cooperativos ${ }^{5}$. Sin embargo estos planteamientos se encuentran con la típica dificultad de poder separar la personalidad previa de la resultante de la misma adicción, con lo que las diferencias que se puedan hallar siempre quedan con el interrogante de hasta que punto han estado producidas por el consumo abusivo de alcohol o eran un factor previo de riesgo de éste consumo.

Otro planteamiento busca estudiar las diferencias entre los adictos a distintas substancias ${ }^{6}$. Por una parte su conocimiento podría servir para desarrollar tratamientos específicos para cada adicción en función de sus características psicológicas diferenciales. Por otra parte también serviría de base a las hipótesis de una supuesta elección de sustancias en función de las características de personalidad previas.

Sin embargo las posibles diferencias halladas tienden a estar "contaminadas" por la heterogeneidad de las muestras, que tienen procedencias y biografías muchas veces distintas y poco comparables. Cuando se hallan diferencias, como la mayor asertividad y mejor competencia social de cocainómanos respecto de heroinómanos, o la mayor improvisación de éstos ${ }^{6}$, son diferencias sutiles y mucho menores que las grandes similitudes que presentan.

Por todo ello la meta que nos planteamos es más prosaica y no pretende buscar diferencias conceptua- les o teóricas entre grupos que son en sí diferentes, ni tampoco hipotetizar sobre los factores de la personalidad que pueden ser de riesgo para desarrollar una adicción. La pretensión es encontrar aspectos diferenciales concretos que nos ayuden en la práctica clínica diaria con enfermos que tienen en común su alcoholismo. Estas diferencias individuales son las que pueden explicar el que a pesar de la existencia de técnicas psicológicas de tratamiento de la dependencia alcohólica que han demostrado su eficacia ${ }^{15}$, no se ha podido encontrar cuál es superior a las demás, lo que sugiere que personas distintas se han de beneficiar de abordajes psicológicos diferentes ${ }^{16}$.

El objetivo de este estudio es pues conocer las diferencias en las características de personalidad, medidas con el cuestionario de personalidad Temperament and Character Inventory (TCl), entre el colectivo de pacientes alcohólicos en función de que presenten o no, un abuso de benzodiazepinas (BZD) o un trastorno de la personalidad (TP). Estos factores diferenciales podrían ser considerados entonces de mal o buen pronóstico, y podrían convertirse en dianas en las que centrar nuestros esfuerzos psicoterapéuticos.

Esta pretensión parte del reconocimiento de la mayor gravedad y dificultades terapéuticas que comporta el manejo de los pacientes alcohólicos dependientes también a BZD y/o con TP, debido en parte a la existencia de elevados niveles de ansiedad y de problemática psiquiátrica más grave y duradera.

\section{MATERIAL Y MÉTODO}

Se administró el cuestionario $\mathrm{TCl}$ a 218 pacientes ingresados consecutivamente para desintoxicación del alcohol, y cuando fue necesario también de otras drogas, en una Unidad Hospitalaria de Desintoxicación (UHD). Criterios de inclusión: existencia de una dependencia al alcohol activa según criterios DSM-IV como motivo principal del ingreso. Criterios de exclusión: ser un reingreso para desintoxicación o tener una incapacidad física, psiquiátrica o intelectual para responder el cuestionario.

Todos los pacientes fueron evaluados rutinariamente por un equipo formado por psiquiatra, psicólogo y asistente social. Los ingresos de desintoxicación duraron una semana, durante la cual se realizó también un tratamiento psicoterapéutico con visitas y controles toxicológicos en orina diarios, con el objetivo de lograr una conciencia de dependencia, motivación para el mantenimiento de la abstinencia y habilidades para lograrla con posterioridad al alta hospitalaria.

Se exploró la existencia de trastornos de la personalidad siguiendo criterios del eje II del DSM-IV y de deterioro neuropsicológico mediante vías diversas 
en función de la situación del enfermo: en algunos casos se usaron técnicas de neuroimagen, o baterías neuropsicológicas. La existencia de síntomas clínicos indicativos, como déficits objetivables de memoria, fabulaciones, cambios inapropiados de humor, inconsistencias y contradicciones en la elaboración de la anamnesis, dificultades de comprensión y cumplimiento de las indicaciones terapéuticas o una actitud de negación patológica de los problemas alcohólicos, orientó en la dirección de la existencia de deterioro cognitivo.

En general el examen de la biografía del enfermo fue una fuente de evidencia de la afectación cognitiva. En ella, además de las dificultades para una conducta diaria integrada en su medio, o los cambios en el comportamiento, fue frecuente encontrar un historial de deliriums por abstinencia, encefalopatías hepáticas o neuropatías.

Se analizaron las diferencias en las escalas del TCl entre pacientes con o sin dependencia a BZD, y entre pacientes con o sin TP.

\section{Instrumento}

El TCl (Temperament and Character Inventory) es un inventario de rasgos de personalidad ${ }^{17}$ autoadministrado, validado en nuestro entorno ${ }^{18}$, que ya ha sido utilizado para estudiar diversas tipologías de pacientes alcohólicos ${ }^{19}$. Mide siete dimensiones de la personalidad siguiendo una teoría que desarrolla un modelo psicobiológico y dimensional que integra el papel de ciertos neurotransmisores (serotonina, dopamina, neurotonina) en la regulación de la conducta.

Estas dimensiones se dividen en cuatro escalas de Temperamento (Evitación del daño, Búsqueda de Novedad, Dependencia de la Recompensa y Persistencia) y tres de Carácter (Auto-dirección, Cooperación y Auto-trascendencia).

\section{Análisis estadístico}

Descripción de las variables evaluadas en la muestra: frecuencias y porcentajes en las variables cualitativas (sexo, abuso/dependencia de BZD, trastornos de la personalidad) y medidas de tendencia central en las variables cuantitativas.

Para la comparación de los porcentajes de cada patrón de consumo de BZD, del abuso de éstas y de la presencia de trastornos de la personalidad (TP) entre ambos sexos, se utilizó la prueba de $\chi^{2}$ (chi cuadrado).

La prueba de la t de Student para grupos independientes se usó para comparar las puntuaciones del $\mathrm{TCl}$ entre ambos sexos y entre pacientes con o sin TP o abuso/dependencia de BZD.

\section{RESULTADOS}

$156(71,6 \%)$ de los pacientes de la muestra estudiada eran varones. La edad media fue de 46,3 $\pm 8,8$ años, sin diferencias significativas entre ambos sexos. La Tabla 1 muestra los diagnósticos referidos al consumo de BZD y sus diferencias entre sexos.

Existían diferencias entre ambos sexos en cuanto al porcentaje de consumo abusivo de BZD $(21,8 \%$ de los hombres y $54,8 \%$ de las mujeres; $\mathrm{Chi}^{2}=22,5$; $\mathrm{p}<0,0001)$ y el porcentaje de trastornos de la personalidad $(9,6 \%$ de los hombres y $19,4 \%$ de las mujeres; $\left.\mathrm{Chi}^{2}=3,8 ; \mathrm{p}=0,049\right)$.

$27(12,4 \%)$ pacientes recibieron un diagnóstico de trastorno de la personalidad (TP), de los que 12 eran inespecíficos (con criterios de más de un TP) y el resto del cluster $B$ (5 histriónicos, 6 antisociales, 2 narcisistas y 2 límites).

Tabla 1. Patrón de abuso/dependencia de BZD. Diferencias entre sexos.

\begin{tabular}{|l|c|c|c|}
\hline Patrón de consumo de BZD & & $\begin{array}{c}\text { Hombre } \\
\mathbf{N = 1 5 6}\end{array}$ & $\begin{array}{c}\text { Mujer } \\
\text { N=62 }\end{array}$ \\
\hline Abuso episódico & $38(17,4 \%)$ & $20(12,8 \%)$ & $18(29 \%)$ \\
\hline Abuso continuo & $9(4,1 \%)$ & $6(3,8 \%)$ & $3(4,8 \%)$ \\
\hline Dependencia episódica & $3(1,4 \%)$ & $1(0,6 \%)$ & $2(3,2 \%)$ \\
\hline Dependencia continua & $18(8,3 \%)$ & $7(4,5 \%)$ & $11(17,7 \%)$ \\
\hline Abuso en remisión & $1(0,5 \%)$ & $1(0,6 \%)$ & 0 \\
\hline No consumo de BZD & $149(68,3 \%)$ & $121(77,6 \%)$ & $28(45,2 \%)$ \\
\hline Total & $218(100 \%)$ & $100 \%$ & $100 \%$ \\
\hline
\end{tabular}

BZD: benzodiacepinas. 
Tabla 2. Puntuaciones medias y desviaciones típicas en las notas T delTCI para toda la muestra y comparación de puntuaciones entre pacientes en función del sexo.

\begin{tabular}{|l|c|c|c|}
\hline Escalas básicas TCI & Notas T & Hombre & Mujer \\
\hline HA (Evitación del daño) & $61,6 \pm 9,3$ & $\mathbf{6 0 , 7 \pm 9}$ & $\mathbf{6 3 , 8 \pm 9 *}$ \\
\hline NS (Búsqueda de novedad) & $52,3 \pm 8,8$ & $52,4 \pm 8$ & $52,8 \pm 9$ \\
\hline RD (Dependencia de la Recompensa) & $49,9 \pm 7,8$ & $\mathbf{4 9 , 1 \pm 7}$ & $\mathbf{5 1 , 9 \pm 8 *}$ \\
\hline P (Persistencia) & $45,2 \pm 9,5$ & $45,1 \pm 9$ & $45,4 \pm 8$ \\
\hline S (Autodirección) & $41 \pm 9,7$ & $40,8 \pm 10$ & $41,3 \pm 9$ \\
\hline C (Cooperación) & $46,1 \pm 8,1$ & $\mathbf{4 5 , 1 \pm 8}$ & $\mathbf{4 8 , 6 \pm 7 * *}$ \\
\hline ST (Auto-trascendencia) & $43,4 \pm 9,3$ & $43 \pm 9$ & $44,6 \pm 9$ \\
\hline
\end{tabular}

${ }^{*} p<0,05 ;{ }^{* *} p<0,005$.

Tabla 3. Puntuaciones medias y desviaciones típicas en las notas T del TCI en función de la existencia de trastornos de la personalidad y abuso/dependencia de BZD.

\begin{tabular}{|l|c|c|c|c|}
\hline Escalas básicas TCI & $\begin{array}{c}\text { BZD } \\
\text { N= 68 }\end{array}$ & $\begin{array}{c}\text { No BZD } \\
\mathbf{N = 1 5 0}\end{array}$ & $\begin{array}{c}\text { TP } \\
\mathbf{N = 2 7}\end{array}$ & $\begin{array}{c}\text { Sin TP } \\
\text { N= 191 }\end{array}$ \\
\hline HA (Evitación del daño) & $\mathbf{6 5 , 6 \pm 9}$ & $\mathbf{5 9 , 8 \pm 8 * *}$ & $62 \pm 9$ & $61,5 \pm 9$ \\
\hline NS (Búsqueda de novedad) & $53,3 \pm 8$ & $52,1 \pm 8$ & $\mathbf{5 5 , 6 \pm 7}$ & $\mathbf{5 2 \pm 8 *}$ \\
\hline RD (Dependencia de la Recompensa) & $48,9 \pm 8$ & $50,3 \pm 7$ & $50 \pm 8$ & $49,8 \pm 7$ \\
\hline P (Persistencia) & $46,5 \pm 8$ & $44,6 \pm 9$ & $50,3 \pm 7$ & $44,5 \pm 9$ \\
\hline S (Autodirección) & $\mathbf{3 8 , 9 \pm 9}$ & $\mathbf{4 1 , 9 \pm 9 *}$ & $38 \pm 9$ & $41,4 \pm 9$ \\
\hline C (Cooperación) & $44,6 \pm 9$ & $46,8 \pm 7$ & $42,3 \pm 9$ & $46,6 \pm 7 *$ \\
\hline ST (Auto-trascendencia) & $42,1 \pm 9$ & $44 \pm 9$ & $45,4 \pm 9$ & $43,1 \pm 9$ \\
\hline
\end{tabular}

${ }^{*} p<0,05 ;{ }^{*} p<0,005$.

TP: Trastorno de la personalidad. BZD: Abuso o dependencia de benzodiacepinas.

La Tabla 2 muestra las puntuaciones de las escalas del TCl y las diferencias en función del sexo. La Tabla 3 muestra las puntuaciones $\mathrm{TCl}$ en función de la existencia de un TP o de un abuso/dependencia de BZD. No había diferencias significativas en ninguna de las puntuaciones de las escalas TCl según el tipo de TP.

\section{DISCUSIÓN}

El hallazgo de que las mujeres alcohólicas son consumidoras de BZD más a menudo que los hombres y tienen con mayor frecuencia un diagnóstico de TP, coincide con estudios previos ${ }^{20,21,22}$ aunque ésta cuestión no es el objetivo del estudio y puede tener dificultades de generalización, al excluir nuestros criterios de selección a pacientes con dificultades para responder al TCl, lo que podría sesgar a subgrupos específicos, como mujeres que por su nivel de consumo y estado de ansiedad no pueden completar las pruebas.

La mayoría de TP diagnosticados lo son del cluster B. El resto de TP cumplen criterios inespecíficos, la mayor parte de veces también con algún síntoma del cluster B.

Las mujeres se caracterizan por una mayor evitación del daño, ser más dependientes de la recompensa y cooperar más que los hombres, según las escalas del $\mathrm{TCl}$.

Estos rasgos corresponden a personas más ansiosas, de base más neurótica, más influenciables en su conducta por gratificaciones externas y con mayor capacidad para beneficiarse de la experiencia de otras personas con las que estén trabajando hacia metas comunes. Estos datos concuerdan con los hallazgos que muestran que las mujeres tienen un mejor pronóstico del tratamiento alcohológico, especialmente utilizando técnicas grupales ${ }^{23}$.

También sugieren que el seguimiento a largo plazo de estos pacientes que ingresan para desintoxicación será distinto para hombres y mujeres, con una mejor adaptación para ellas. En un estudio de seguimiento previo $^{24}$ no hallamos una mejor evolución global, pero la revisión minuciosa de los datos reveló después que aunque se cronifican más que los hombres lo es a costa de abandonar mucho menos el tratamiento, 
punto que concuerda con otro hallazgo de un estudio de seguimiento comparando entre hombres y mujeres alcohólicos con tentativas autolíticas ${ }^{25}$, en el que éstas muestran porcentajes de abstinencia a los 5 años mucho mayores, simultáneamente a una mayor adherencia terapéutica.

Según el TCl los pacientes con abuso/dependencia de BZD evitan más el daño y tienen un lugar de control más externo (menor puntuación en autodirección).

La primera característica podría ser favorable para el mantenimiento de la abstinencia, pues supone la utilización de estrategias de detección de riesgos y su evitación, pero aunque en las mujeres puede ser una ventaja, en este grupo de pacientes está aún más elevada y puede hacer la conducta inhibida en lugar de precavida.

Por otro lado la menor autodirección supone menos madurez y autosuficiencia. Son personas con un lugar de control más externo, más fácilmente influenciables y cuya conducta es más voluble e impredecible, ligada a las circunstancias, impulsos o estímulos externos. Ello concuerda con la impresión clínica de que los pacientes con abuso/dependencia de BZD tienen menor capacidad para dirigir la conducta hacia metas escogidas voluntaria e individualmente.

Ello implica que los esfuerzos terapéuticos en pacientes dependientes a BZD deben dirigirse hacia el estimulo de su independencia, lo que supone que en lugar de protegerles y evitar que corran riesgos, se les debe enseñar a hacer ensayos o enfrentamientos "controlados" de daños o peligros como un proceso de experimentación y maduración personal.

Los pacientes con TP se caracterizan por una mayor búsqueda de novedad y menor cooperación.

La búsqueda de novedad es un rasgo que mide la exploración, desinhibición, impulsividad y el grado de control o excitabilidad del individuo. Por ello los resultados son coherentes con el hecho de que la mayoría de pacientes con TP lo son del cluster B, en que predominan las dificultades en el control de los impulsos.

La cooperación se caracteriza por la empatía, la cordialidad, el altruismo y el respeto de los demás. En los pacientes alcohólicos con TP existe un proceso de aislamiento social mayor que en los que no tienen TP. Éste probablemente se ha generado mayoritariamente con anterioridad al consumo abusivo de alcohol, pero también es posible que en personas con TP el efecto del alcohol sea más catastrófico a nivel de romper los vínculos emocionales con los demás.

Éstos datos confirman al clínico el predominio de los trastornos de la personalidad antisociales e histriónicos, junto con una serie de casos más abigarrados, pero en los que también coexisten las características de estos trastornos. Le indican también la necesidad de trabajar en dos dianas terapéuticas principales: la desinhibición y control de los impulsos y la empatía y respeto a los demás.

En conclusión, las características encontradas con el $\mathrm{TCl}$ son coherentes con las dificultades de estos pacientes durante el tratamiento y con las impresiones clínicas: los pacientes alcohólicos y dependientes a BZD son más inseguros, ansiosos, evitadores y con una conducta muy voluble e influenciable por estímulos externos, mientras que los pacientes alcohólicos con TP acostumbran a ser más impulsivos y menos cooperadores. Por todo ello el TCl se revela un instrumento útil en la comprensión, evaluación e identificación de los pacientes alcohólicos, así como en la visualización de objetivos terapéuticos concretos para intentar modular o controlar la expresión de su personalidad.

\section{REFERENCIAS}

1. Merikangas K, Stevens DE. Substance abuse among women: Familial factors and comorbidity. In: Wetherington $C L$, Roman $A B$, editors. Drug Addiction Research and the Health of Women. Bethesda, MD: National Institute on Drug Abuse, 1998: 245-269.

2. Wittchen HU, Nelson CB, Lachner G. Prevalence of mental disorders and psychosocial impairments in adolescents and young adults. Psychol Med 1998; 28:109-126.

3. Haro G, Mateu C, Martínez-Raga J, Valderrama JC, Castellano M, Cervera G. The role of personality disorders on drug dependence treatment outcomes following inpatient detoxification. European Psychiatry 2004; 19: 187-192.

4. Margolese HC, Malchy L, Negrete JC, Tempier R, Gill K. Drug and alcohol use among patients with schizophrenia and related psychoses: Levels and consequences. Schizophr Res 2004; 67: 157-166.

5. Mateos J, Mateos M. Rasgos diferenciales del temperamento y el carácter en un grupo de alcohólicos vs población general. Adicciones 2005; 17: 325-335.

6. Barron E, Mateos M, Martínez I. Temperamento y carácter en adictos a cocaína y a opiáceos. Adicciones 2004; 16: 287-294.

7. Vaillant $\mathrm{G}$. The natural history of alcoholism. Cambridge: Harvard University Press; 1983.

8. Vaillant GE. What can long-term follow-up teach us about relapse and prevention of relapse in addiction? Br J Addict 1988; 83: 1147-57.

9. Vaillant GE. Natural history of male psychological health: VIII. Antecedents of alcoholism and "orality". Am J Psychiatry 1980; 137: 181-6. 
10. Grekin ER, Sher KJ, Wood PK. Personality and substance dependence symptoms: modeling substance-specific traits. Psychol Addict Behav 2006; 20: 415-24

11. Kessler RC. Impact of substance abuse on the diagnosis, course, and treatment of mood disorders. The epidemiology of dual diagnosis. Biol Psychiatry 2004; 56: 730-7.

12. Rhee SH, Hewitt JK, Young SE, Corley RP, Crowley TJ, Stallings MC. Genetic and environmental influences on substance initiation, use, and problem use in adolescents. Arch Gen Psychiatry 2003; 60: 1256-64.

13. McGue M. The behavioral genetics of alcoholism. Curr Dir Psychol Sci 1999; 8: 109-115.

14. Kendler KS, Jacobson KC, Prescott CA, Neale MC. Specificity of genetic and environmental risk factors for use and abuse/dependence of cannabis, cocaine, hallucinogens, sedatives, stimulants, and opiates in male twins. Am J Psychiatry 2003; 160: 687-95

15. Anton RF, O'Malley SS, Ciraulo DA, Cisler RA, Couper D, Donovan DM, et al. Combined pharmacotherapies and behavioral interventions for alcohol dependence: the COMBINE study: a randomized controlled trial. JAMA 2006; 295: 2003-17.

16. Karno MP, Longabaugh R. What do we know? Process analysis and the search for a better understanding of Project MATCH's anger-by-treatment matching effect. J Stud Alcohol 2004; 65: 501-12.

17. Cloninger CR, Svrakic DM, Przybeck TR. A psychobiological model of temperament and character. Arch Gen Psychiatry 1993; 50: 975-90.

18. Gutierrez F, Torrens M, Boget T, Martin-Santos R, Sangorrin J, Perez G, et al. Psychometric properties of the Temperament and Character Inventory (TCI) questionnaire in a Spanish psychiatric population. Acta Psychiatr Scand 2001; 103: 143-7.

19. Basiaux P, Le Bon O, Dramaix M, Massat I, Souery $D$, Mendlewicz J, et al. Temperament and Character Inventory ( $\mathrm{TCl}$ ) personality profile an sub-typing in alcoholic patients: a controlled study. Alcohol Alcohol 2001; 36: 584-7.

20. Nomura K, Nakao M, Sato M, Yano E. Regular prescriptions for benzodiazepines: a cross-sectional study of outpatients at a university hospital. Intern Med 2006; 45: 1279-83.

21. Petrovic $M$, Vandierendonck $A$, Mariman $A$, van Maele G, Afschrift M, Pevernagie D. Personality traits and socio-epidemiological status of hospitalised elderly benzodiazepine users. Int J Geriatr Psychiatry 2002; 17: 733-8.

22. Hellerstein DJ, Flansaas DM, Zweben-Howland LD, Samstag LW. A clinical profile of patients affected by state regulation of benzodiazepine prescriptions. Hosp Community Psychiatry 1994; 45: 362-5.

23. Monras M, Freixa N, Ortega L, Lligoña A, Mondon S, Gual A. Eficacia de la terapia de grupo para alcohólicos. Resultados de un ensayo clínico controlado. Medicina Clínica (Barc) 2000; 115: 126-131.

24. Monras M, Garcia S, Torres M, Gual A, Ortega L. El ingreso hospitalario en el tratamiento de los alcohólicos cronificados. Adicciones 1998; 10: 151-160.

25. Monras M. Seguimiento a 5 años de alcohólicos con antecedentes de tentativas suicidas. Adicciones 1995; 7: 479-494. 